\title{
Rapunzel syndrome in a seven year old female
}

\author{
Anand Gupta, Jaswinder Kaur, Nishant Wadhwa \\ Sir Ganga Ram Hospital, Division of Pediatric Gastroenterology, Hepatology and Liver Transplant, New Delhi, India. \\ E-mail: drnishantwadhwa@gmail.com \\ Received: 21th March 2017, Accepted: 4th May 2017
}

\begin{abstract}
SUMMARY: Gupta A, Kaur J, Wadhwa N. Rapunzel syndrome in a seven year old female. Turk J Pediatr 2017; 59: 598-600.

Rapunzel syndrome is characterized by gastric trichobezoar with extension beyond pylorus. Seven year old female presented with chronic pain abdomen and loss of appetite. Upper gastrointestinal (UGI) endoscopy showed large trichobezoar in the stomach with its tail extending into proximal duodenum. Child underwent exploratory laparotomy and a large trichobezoar measuring $15 \mathrm{~cm}$ was removed. Psychiatric opinion revealed that the child was suffering from 'trichotillomania'; an obsessive compulsive disorder. UGI endoscopy is the preferred modality for diagnosis, as it delineates the mass and its extent. Small bezoars can be extracted via endoscopy using various disintegrating techniques but large bezoars need surgical management.
\end{abstract}

Key words: Rapunzel, trichobezoar, trichotillomania.

Rapunzel syndrome is characterized by a gastric trichobezoar extending into the small bowel via pylorus. ${ }^{1,2}$ The term comes from a story written by the Grimm brothers in 1812 about Rapunzel who was a long haired maiden. She lowered her tresses to allow her prince charming to climb up to her prison and rescue her. Rapunzel syndrome was first reported by Vaughan in $1968 .{ }^{3}$ Since then; few cases have been reported in literature so far. It is a very rare manifestation of trichobezoar, in which strands of swallowed hair extends into the small intestine via pylorus. Trichotillomania is an obsessive compulsive disorder characterized by excessive pulling of hair and it is a disease of adolescents and adults. It is rather rare in children.

Here we present a seven year old girl with Rapunzel syndrome and underlying trichotillomania who presented with pain abdomen and reduced oral intake.

\section{Case Report}

A seven year old girl presented with complaints of abdominal pain from past 5 months. Pain was non localized, mild to moderate in intensity, intermittent and increased after food intake. There was also history of loss of appetite resulting in reduced oral intake and significant weight loss in past 3 months. There was no history of recurrent fever, abdominal distension, vomiting, constipation, diarrhea or blood in stools.

On examination, child was hemodynamically stable. She had mild pallor, and on per abdomen examination, a firm mass was palpable in the left hypochondrium and epigastrium. The mass was mobile and fingers could be insinuated under the left costal margin. There was no hepatosplenomegaly or free fluid. The rest of the systemic examination was unremarkable. Investigations revealed normal blood counts (hemoglobin $10.7 \mathrm{~g} / \mathrm{dl}$, platelet count 448,000/ $\mathrm{mm}^{3}$, white blood cell $7,200 / \mathrm{mm}^{3}$ ). Liver and renal function tests were normal. USG abdomen revealed large rounded mass with distal acoustic shadowing in epigastrium and left hypochondrium. CT scan was suggestive of large mass in fundus and body of the stomach with tail extending into the proximal duodenum. Upper gastrointestinal (UGI) endoscopy was done which revealed large trichohezoar in gastric fundus, body and antrum and its tail was extending into the duodenum. There were erosions in the antrum, fundus and second part of the duodenum.

As the mass was large exploratory laparotomy was planned and a large trichobezoar measuring 

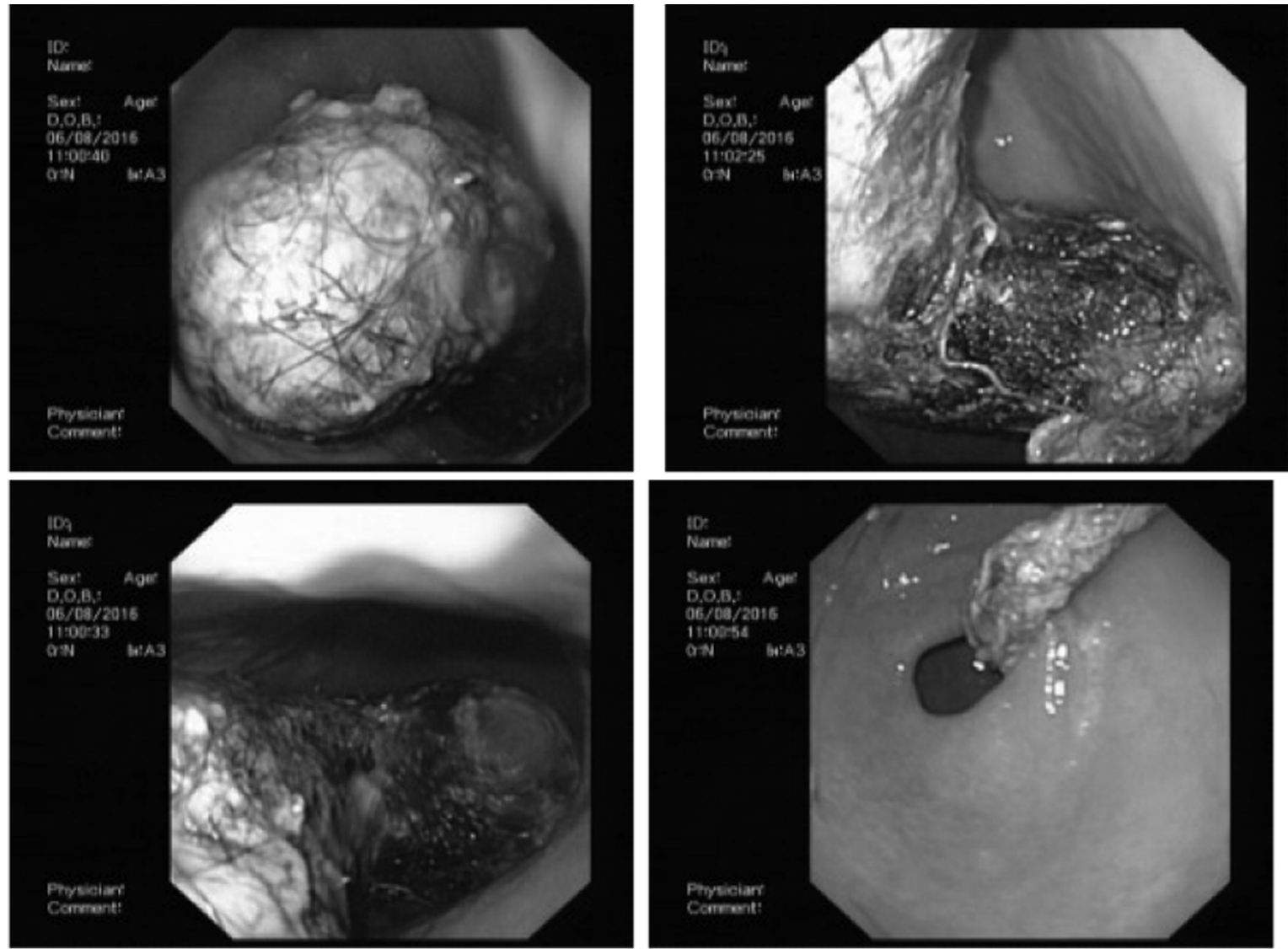

Fig.1. Endoscopic images of bezoar, large bezoar seen occupying stomach and migrating through pylorus.

approximately $15 \mathrm{~cm}$ which had taken the shape of stomach was removed. On detailed evaluation, history of pulling out of hairs was elicited from the mother and child was noted to have sparse hair in both the temporal regions. Psychiatry consultation was taken and child was diagnosed to have Trichotillomania and was started with cognitive behavioural therapy. Child remained stable throughout hospital stay and was discharged on 5th postoperative day and continued with behavioral therapy at home. Informed consent was taken from the parents of the child to report the case.

\section{Discussion}

Bezoars are concretions of foreign material in the gastrointestinal tract. They have been classified into phytobezoars, pharmacobezoars, trichobezoars, lactobezoars according to substance ingested. ${ }^{4}$ Trichobezoar is a ball of swallowed hair that collects in the stomach and fails to pass through the intestines. They are confined to stomach in most of the cases.

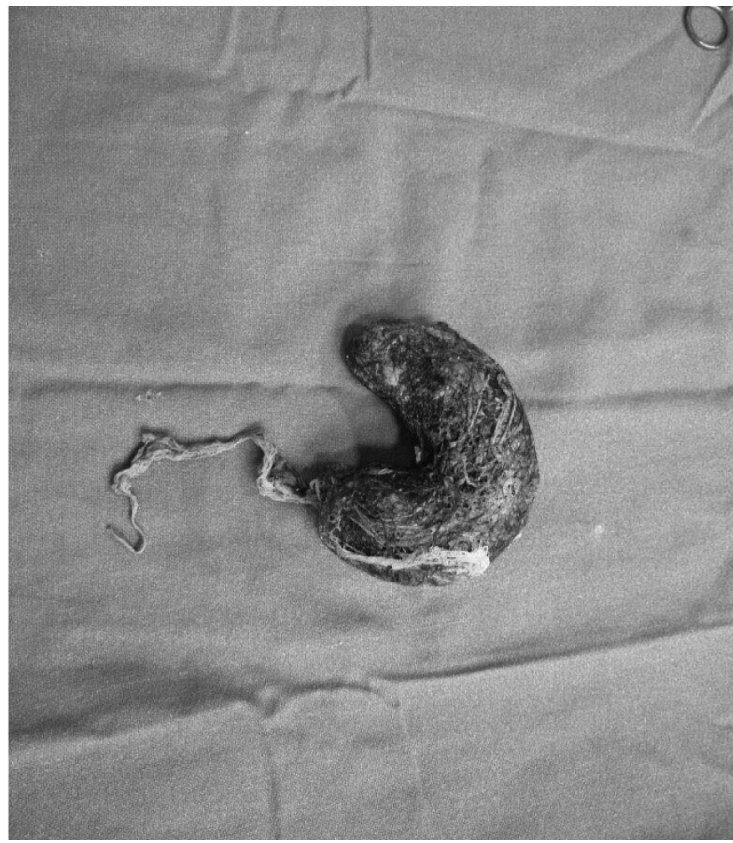

Fig. 2. Specimen of trichobezoar. It has taken the shape of stomach and the strands are seen which have migrated to small intestine through pylorus. 
However, rarely there is contiguous extension of trichobezoar through the pylorus into the small intestine. Such condition is termed as Rapunzel syndrome. It is a very rare entity in pediatric population, with only few cases described in literature so far. The entity most commonly affects females aged $<30$ years, youngest reported case being 6 months. ${ }^{5}$ Trichobezoar is commonly a secondary manifestation in young females with psychiatric disorders. ${ }^{6}$

Trichobezoar may present with pain abdomen, vomiting, intestinal obstruction, and pancreatitis. Slow growing trichobezoar may lead to obstruction, ulceration, perforation or hemorrhage in corresponding part of bowel if left untreated. ${ }^{1,2}$

Trichobezoar have been reported in pediatric age with iron deficiency anemia which leads to PICA, therefore leading to ingestion of nonedible things. A rare case of celiac disease with trichobezoar has been reported in literature. ${ }^{7}$ $\mathrm{X}$ - ray and ultrasonography is not diagnostic, rather, computed tomography scan and upper gastrointestinal endoscopy are the diagnostic modalities of choice. ${ }^{8}$ Treatment is almost always surgical, small bezoars can be extracted via endoscopy using various disintegrating techniques. ${ }^{9}$ However, exploratory laparotomy and excision is the favoured approach. Large bezoars $(>20 \mathrm{~cm})$ are usually removed via surgical approach (laparotomy or laparoscopy). ${ }^{10}$ Routine endoscopy, ultrasonography may be used for detection of recurrence. Our case was seven year old girl; there was history suggestive of trichotillomania. The diagnosis was made by history, physical examination, CT scan and UGI endoscopy. The bezoar was removed by exploratory laparotomy.

Rapunzel syndrome is extremely rare condition in children. High index of suspicion is required for diagnosis. CT scan, UGI endoscopy is crucial for diagnosis. Management is surgical for such cases.

\section{REFERENCES}

1. Meyer-Rochow GY, Grunewald B. Laparoscopic removal of a gastric trichobezoar in a pregnant woman. Surg Laparosc Endosc Percutan Tech 2007; 17: 129-132.

2. Mehta P, Bhutani R. The Rapunzel syndrome: Is it an Asian problem? (case report and review of literature) Eur J Gastroenterol Hepatol 2009; 21: 937-940.

3. Vaughan ED Jr, Sawyer JL, Scott HW Jr. The Rapunzel syndrome. An unusual complication of intestinal bezoar. Surgery 1908; 63: 339-343.

4. Grosfeld JL, Schreiner RL, Franken EA, et al. The changing patterns of gastrointestinal bezoar in infants and children. Surgery 1980; 88: 425-432.

5. Screiber H, Filston HC. Obstructive jaundice due to gastric trichobezoar. J Pediatr Surg 1976; 11: 103-104.

6. Phillips MR, Zaheer S, Drugas GT. Gastric trichobeazoar; case report and literature review. Mayo Clin Proc 1998; 73: 653-656.

7. Inaki I, Carlos T, Juan CV. A trichobezoar in a child with undiagnosed celiac disease: A case report. World J Gastroenterol 2014; 20: 1357-1360.

8. Alsa Fwah S, Alzein M. Small bowel obstruction due to trichobezoar: role of upper endoscopy in diagnosis. Gastrointest Endosc 2000; 52: 784-786.

9. Wang YG, Seitz U, CIZL, Soehendra N, Qiao XA. Endoscopic management of huge bezoars. Endoscopy 1998; 30: 371-374.

10. Groenwald CB, Smoot RL, Farley DR. A football-sized gastric mass in healthy teen. Contemp Surg 2006; 62: 531-534. 